\title{
On Count-to-Infinity Induced Forwarding Loops in Ethernet Networks
}

\author{
Khaled Elmeleegy, Alan L. Cox, T. S. Eugene Ng \\ Department of Computer Science \\ Rice University
}

\begin{abstract}
Ethernet's high performance, low cost and ubiquity have made it the dominant networking technology for many application domains. Unfortunately, its distributed forwarding topology computation protocol - the Rapid Spanning Tree Protocol (RSTP) - can suffer from a classic "count-to-infinity" problem that may lead to a forwarding loop under certain network failures. The consequences are serious. During the period of "count-to-infinity", which can last tens of seconds even in a small network, the network can become highly congested by packets that persist in cycles in the network, even packet forwarding can fail as the forwarding tables are polluted. In this paper, we explain the origin of this problem in detail and study its behavior. We find that simply tuning RSTP's parameter settings cannot adequately address the fundamental problem with "count-toinfinity". We propose a simple and effective solution called RSTP with Epochs. This approach uses epochs of sequence numbers in protocol messages to eliminate stale protocol information in the network and allows the forwarding topology to recover in merely one round-trip time across the network.
\end{abstract}

\section{INTRODUCTION}

Ethernet $^{1}$ is the dominant networking technology in environments ranging from home networks, office networks, data center networks, campus networks, and is becoming more popular in metropolitan-area networks as well. By far the most important reasons for Ethernet's dominance are its high performance-to-cost ratio and its ubiquity. Virtually all computer systems today have an Ethernet interface built in. Ethernet is also fully plug-and-play, requiring no error-prone manual configuration. Moreover, because Ethernet is a layer 2 technology, many layer 3 protocols can easily co-exist on Ethernet networks.

Even though Ethernet has all of these compelling benefits, mission-critical applications also demand high network dependability. The dependability of Ethernet in the face of partial network failure is the focus of this study.

Ethernet has a unique combination of features enabling plug-and-play operation. First, Ethernet requires no manual interface address configuration for switches or end systems.

This research was sponsored by the NSF under CAREER Award CNS0448546 NeTS Award CNS-0435425, by the Texas Advanced Research Program under grant No.003604-0078-2003, and by Cisco Systems, Inc. Views and conclusions contained in this document are those of the authors and should not be interpreted as representing the official policies, either expressed or implied, of NSF, the state of Texas, Cisco Systems, Inc., or the U.S. government.

${ }^{1}$ In this paper, Ethernet always refers to the modern switched network technology as opposed to the legacy broadcast network technology.
Ethernet addresses are simple globally unique identifiers, usually assigned by hardware manufacturers, that do not have any special hierarchical structure for packet forwarding. To deliver a packet from a source to an unknown destination address, Ethernet switches flood the packet throughout the network to ensure it reaches its destination. However, flooding is highly inefficient. Fortunately, an Ethernet switch can observe the flooding of a packet to determine the switch port at which a packet from a particular source address $S$ arrives. This switch port then becomes the outgoing port for packets destined for $S$ and so no flooding is required to deliver future packets to $S$. Thus, an Ethernet network dynamically discovers the topological locations of interface addresses and dynamically builds packet forwarding tables accordingly. This mechanism is called address learning.

To support the flooding of packets for unknown destinations and address learning, an Ethernet network also dynamically and distributedly computes a cycle-free active forwarding topology using the Rapid Spanning Tree Protocol (RSTP). This active forwarding tree is a logical overlay on the underlying physical topology. Cycles in the underlying physical topology provide redundancy in the event of a link or switch failure. It is critical to not allow cycles in the active forwarding topology. Otherwise, first of all, flooded packets will persist indefinitely in the network cycle causing congestion. Secondly, address learning will not function correctly because a switch may receive packets from a source $S$ via multiple switch ports, making it impossible to build the forwarding table correctly.

The dependability of Ethernet therefore heavily relies on the ability of RSTP to quickly recompute a cycle-free active forwarding topology upon a partial network failure. Some pathological causes for forwarding loops in RSTP have been previously documented by Cisco [2]. However, even under normal operation, RSTP may exhibit a "count-to-infinity" problem which can allow a temporary forwarding cycle to exist in the network for tens of seconds. During this period, network congestion may sharply increase and packets may be forwarded incorrectly. This highly unacceptable behavior was mentioned by Myers et al. [11]. To the best of our knowledge, however, no comprehensive examination of this problem exists in the literature.

This paper presents an in-depth examination of this countto-infinity problem in RSTP and provides a simple yet effective solution to the problem. The contributions of this study include: 
- Identify the count-to-infinity problem with specific protocol details in the IEEE 802.1D (2004) specification.

- Demonstrate the exact conditions under which the countto-infinity problem manifests itself.

- Provide a thorough study of the behavior of the countto-infinity problem under different network topologies and protocol parameter settings. We show that protocol parameter tuning cannot adequately improve the convergence time of RSTP.

- Propose and evaluate a simple yet effective solution that removes the count-to-infinity problem and dramatically improves the convergence time of the spanning tree computation upon failure to roughly one round-trip time across the network.

The rest of this paper is organized as follows. Section II provides an introduction to the RSTP protocol. Section III describes how a temporary forwarding loop can form under RSTP. Section IV describes our solution to this problem, the RSTP with Epochs protocol. Section V evaluates this protocol. Section VI discusses related work. Section VII concludes this paper.

\section{The Rapid Spanning Tree Protocol (RSTP)}

RSTP is the current standard Ethernet spanning tree protocol. The Spanning Tree Protocol (STP) is the predecessor of RSTP. As background, we provide a brief overview of both STP and RSTP.

The spanning tree protocols are link management protocols that are designed to allow for redundancy while preventing loops in the active topology. Redundancy is important for fault tolerance to link or bridge failures. However, having loops in the active topology can result in packets persisting in the network as Ethernet packets do not have a time-to-live field. The Spanning Tree Algorithm (STA) builds a unique spanning tree out of the network of bridges. The tree is rooted at the bridge with the lowest ID in the network and spans all bridges in the network. A path from any bridge to the root bridge is guaranteed to be of minimum cost. Traffic is forwarded along these paths within the tree. Since the active topology is a tree, it is by definition loop free. Redundant links are kept in a standby mode (blocked). The STA enables these standby links whenever it detects some failure or a change in the cost of some tree path motivating a reconfiguration of the tree.

Bridge Protocol Data Units (BPDUs) are used by bridges to exchange information regarding their state. The STA uses the BPDU information to elect the root bridge. Each bridge uses the information conveyed in BPDUs to choose the port which lies on the shortest path to the root bridge (its root port) and the ports that connect it to its children in the spanning tree (its designated ports). The root port is the port that has received the best information for a path to the root. Other ports in the bridge send BPDUs with their path cost to the root to other bridges in the network. Ports that receive inferior information than the one they are sending are chosen to be designated ports. Bridges send a BPDU every HelloTime which acts as a heartbeat. A BPDU has a message age that represents the age of the message and is capped by a MaxAge value, when the message age exceeds the MaxAge value the message gets dropped. Each bridge port caps the number of BPDUs it can transmit every second. It has a counter (TxCount) that keeps track of the transmitted BPDUs, if the counter reaches Transmit Hold Count (TxHoldCount) no more BPDUs can get transmitted during the current second. The counter is decremented by one every second.

A topology change can result in the invalidation of a bridge's learned address location information. This is because a topology change can result in the reconfiguration of the spanning tree which may lead to some network segments to appear as if they have moved from one bridge's perspective. This requires the flushing of the forwarding database that caches stations' locations. STA implements this by making a bridge send a Topology Change (TC) message whenever a port is becoming a part of the active topology, it sends such message on all its ports participating in the active topology. A bridge receiving a TC message forwards it on all its ports participating in the active topology except the one it has received the TC message on. Whenever a bridge sends a TC message on one if its ports, it flushes the cached forwarding information at that port.

The following two sections present the differences between the two spanning tree protocols - the Spanning Tree Protocol (STP) and its successor Rapid spanning Tree Protocol (RSTP) - that are relevant to this paper.

\section{A. Spanning Tree Protocol (STP)}

In the event of a topology change, STP relies on timers before switching ports to the forwarding state. This is to ensure that the new information has been spread across the network. The total waiting time can get up to 50 seconds [2]. This conservative value for the waiting time is to protect against prematurely switching a port to the forwarding state resulting in a forwarding loop. Whenever a bridge gets disconnected from the root bridge, it waits until the information cached at its root port is aged out, then it starts accepting other BPDUs from other bridges to discover another path to the root.

In STP the root bridge sends a hello message every HelloTime. Other bridges relay such messages to their children after adjusting the appropriate fields (ex: message age, path cost, ...). A bridge losing a hello message could be due to a problem anywhere along the path to the root.

\section{B. Rapid Spanning Tree Protocol (RSTP)}

RSTP tries to overcome the shortcomings of STP's long convergence time by introducing few optimizations that intend to reduce the convergence time without affecting the functionality of the protocol. For the purpose of this paper we will present the relevant subset of these optimizations. RSTP relies on a handshake between bridges to transition a designated port into the forwarding state rather than waiting for timers. Unlike in STP where a bridge just forwards the root's BPDU messages, in RSTP every bridge sends a BPDU every HelloTime that acts as a heartbeat indicating the 


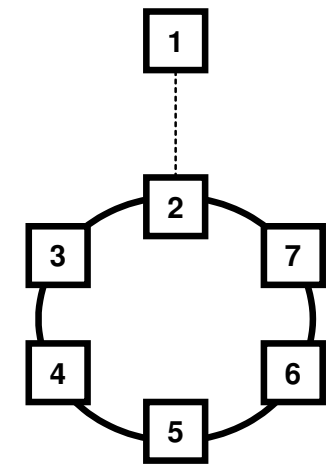

Fig. 1. A simple topology vulnerable to temporary forwarding loop.

liveness of such bridge. This allows for better detection of failed components. If a bridge misses three consecutive BPDU messages on some port, it assumes that the connection has failed and ages out the information at such port. Physical link failures are detected even faster. If a bridge detects failure at its root port, it falls back immediately to an alternate port if it has any. An alternate port is a port with an alternate path to the root bridge [3]. A port is chosen to be either an alternate port or a backup port if it is not the root port and receives superior information than the one it is transmitting. In a switched Ethernet a backup port is a port directly connected to another port on the same bridge. In this paper we are mainly interested in alternate ports. For RSTP a topology change event is when a port that was not forwarding switches to be forwarding.

\section{COUNTING-TO-INFINITY IN RSTP}

This section explains how a temporary forwarding loop may form under the RSTP protocol. A temporary forwarding loop may form when there is a cycle in the physical topology and that this cycle loses connectivity to the root bridge due to a network failure. Figure 1 gives a simple example of a vulnerable topology. The path between bridge 1 (the root) and bridge 2 does not have to be a direct link. A failure in this path can result in a count-to-infinity situation in RSTP that may create a temporary forwarding loop.

\section{A. Counting-to-Infinity: An Example}

To illustrate this problem we will first give a specific example and relate the behavior to clauses in the IEEE 802.1D (2004) [9] standard. In the next section, a general proof is given.

First we state 8 relevant rules that govern the operation of RSTP. Each rule is identified from the IEEE 802.1D (2004) [9] standard.

1) If a bridge can no longer reach the root bridge via its root port and does not have an alternate port, it declares itself to be the root. (Clause 17.6)

2) A bridge sends out a Bridge Protocol Data Unit (BPDU) immediately after the data it is announcing has changed, e.g. when it believes the root has changed or its cost to the root has changed. (Clause 17.8)

3) A designated port becomes the root port if it receives a superior BPDU to what the bridge had received before. That is, this BPDU announces a better path to the root than via the current root port. (Clauses 17.6 and 17.7)

4) An alternate port is a port with an alternate path to the root bridge. A port gets the alternate port role if the BPDU it is to transmit, conveying the cost to the root through itself, is inferior to the one received from its peer. An alternate port caches the information received in the superior BPDU, subjected to a timeout, so that the information can use later if the alternate port becomes the root port. (Clauses 17.6 and 17.7)

5) Bridges (not only the root bridge) send periodic BPDUs, to guard against packet loss and to assist in detecting failed components. (Clause 17.8)

6) A bridge waits for 3 consecutive missing BPDUs from its designated bridge before assuming it to be dead; this is only if the bridge cannot physically detect its failure. After missing three consecutive BPDUs the cached information at the port is aged out. While waiting for the three heartbeats, the bridge generates and transmits its own BPDUs based on its cached information. (Clause 17.21.23)

7) BPDU M1 is superior to BPDU M2 if (Clause 17.5)

a) M1 is announcing a root with a lower bridge ID than that of $\mathrm{M} 2$, or

b) Both BPDUs are announcing the same root but M1 is announcing a lower cost to get to the root, or

c) Both BPDUs are announcing the same root and the same cost but M1 was lastly transmitted through a bridge with a lower ID than that last transmitting $\mathrm{M} 2$, or

d) Both BPDUs are announcing the same root, the same cost and are transmitted last through the same bridge but M1 was transmitted from a port with lower ID than the one last transmitted M2, or

e) Both BPDUs are announcing the same root, the same cost and both were transmitted last through the same bridge and the same port but M1 was received on a port with a lower ID than the one last received M2.

8) The message age is incremented on receipt, and the information discarded if it exceeds the MaxAge. Thus the number of Bridges the information can traverse is limited. (Clause 17.9)

Now consider the example in Figure 2 showing a network of bridges. A box represents a bridge; the top number in the box is the bridge ID, the lower set of numbers represent the root bridge ID as perceived by the current bridge and the cost to this root. The link costs are all 20 (this value is not important). Figure 2(a) shows the stable active topology at time $t 1$. Figure 2(b) shows the network at time $t 2$ when the link between bridge 1 and 2 dies. Bridge 2 declares itself to 


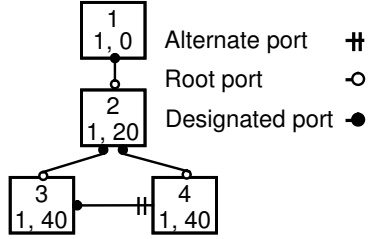

(a) Time $t 1$

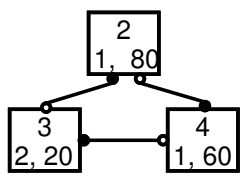

(d) Time $t 4$.

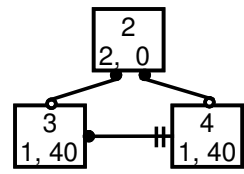

(b) Time t2. Bridge 1 failed.

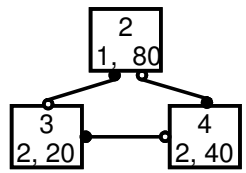

(e) Time $t 5$.

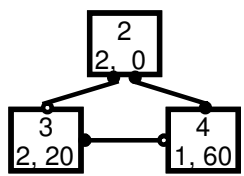

(c) Time $t 3$.

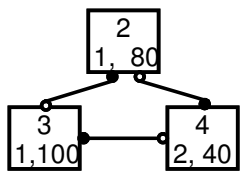

(f) Time $t 6$.

Fig. 2. An example of count to infinity.

be the root since it has no alternate port (rule (1)). Bridge 2 announces to bridges 3 and 4 that it is the root (rule (2)). At time t 3 bridge 3 makes bridge 2 its root as it does not have any alternate port. Bridge 4 however has an alternate port for bridge 1 and incorrectly uses this alternate port as its root port, making bridge 3 its designated bridge (parent in the tree) to the now dead bridge 1 (rule (4)). This is because bridge 4 has no way of knowing that this cached information for the alternate port is stale. At time $t 4$ bridge 4 announces to bridge 2 that it has a path to bridge 1, spreading this stale information and initiating the count-to-infinity (rule (2)). Bridge 2 makes bridge 4 its designated bridge to bridge 1 and updates the cost to bridge 1 to 80 (rule 3). At time t 5 bridge 3 sends a BPDU to bridge 4 saying that bridge 2 is the root. Since bridge 3 is bridge 4 's designated bridge, bridge 4 accepts this information and makes its cost to bridge 2 to be 40 . At time t 6 bridge 2 sends a BPDU to bridge 3 saying that it has a path to bridge 1 . Bridge 3 makes bridge 2 its designated bridge updating its cost to bridge 1 to be 100 . Note that at time $t 3$, bridge 4 's port to bridge 2 is in a temporary blocked state but it becomes forwarding as soon as bridge 2 chooses bridge 4 as its designated bridge and put its port to bridge 3 in a temporary blocked state (time t4). Bridge 2's port to bridge 3 becomes forwarding again at time $t 6$ when bridge 3 confirms that bridge 2 is its designated bridge. Thus at time $t 6$, all ports are forwarding creating a forwarding loop.

When bridge 2 receives the next BPDU from bridge 4 , bridge 2 will transmit BPDUs to reassert its root status. However, the stale information about bridge 1 is still being transmitted by bridge 3 to bridge 4 and continues to go around the cycle in a count-to-infinity situation until this stale information reaches its MaxAge which is supposed to be a timeout with a default value of 20 seconds. However, in reality, the age of a message is incremented by 1 only when it is passed. Thus, a MaxAge of 20 only ensures that the stale information cannot be passed around more than 20 times. As a result, the stale information can actually persist in the network for longer than 20 seconds. (rules 6 and 8).

As the fresh and stale BPDUs cycle around the loop, the bridge ports will eventually reach their TxHoldCount limit.

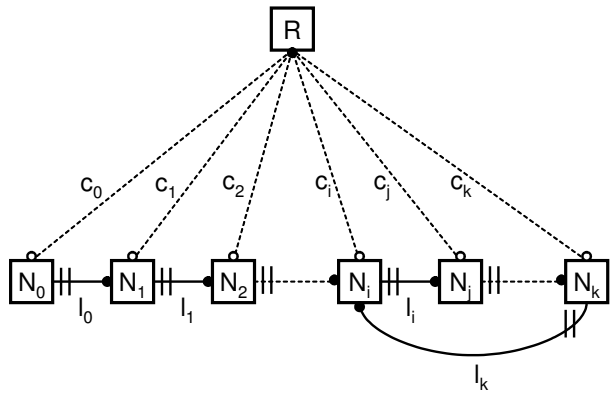

Fig. 3. General network scenario considered in Lemma 1.

Subsequently, a BPDU will only be transmitted by a bridge port when the transmit counter is decremented which happens once a second when the bridge's internal timer ticks. Then depending on when and where the fresh and stale information get stuck in the loop and the clock skew of the bridges, the stale information may catch up with and eliminate the fresh information. When this happens, all the bridges in the loop will believe in the stale information until its MaxAge is reached, all ports in the loop forward packets, thus creating a forwarding loop that lasts until count-to-infinity terminates. On the other hand, the fresh information may catch up with the stale one ending the count-to-infinity.

\section{B. Counting-to-Infinity: The General Case}

We now give a general proof that whenever a network is partitioned, if the partition that does not contain the previous root bridge has a cycle, there exists a race condition that can result in the count-to-infinity behavior which may lead to a temporary forwarding loop. The proof proceeds by first demonstrating that one bridge in the partition without the previous root bridge must declare itself the new root and start transmitting BPDUs. These BPDUs will race around the cycle. Depending on the outcome of the race, count-to-infinity may occur.

Lemma 1: If a network is partitioned, the partition without the previous root bridge must contain a bridge that has no alternate port. 


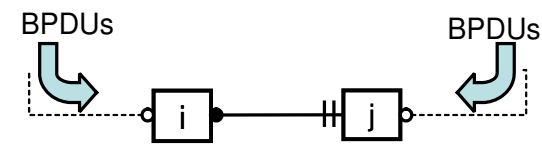

Fig. 4. Illustration of the BPDU Race Condition.

Proof: Consider the general network scenario illustrated in Figure 3. A dotted line represents a network path that may contain unknown intermediate hops. A solid line represents a direct bridge to bridge connection. Before the partition, $R$ is the root bridge in the network. Every bridge $N_{x}$ has a certain shortest path to $R$ with a cost of $c_{x}$. Upon the partition, bridges $N_{0}$ to $N_{k}$ form a partition that has no connectivity to $R$.

The proof is by contradiction. Let us assume that bridges $N_{0}$ to $N_{k}$ all have one or more alternate ports to $R$ immediately after the partition. Consider bridge $N_{0}$. Since $N_{0}$ has at least one alternate port, it must be directly connected to another bridge in the partition, say $N_{1}$, which has an alternate path to $R$ that does not include $N_{0}$. Without loss of generality, assume the BPDU sent by $N_{1}$ is superior than the BPDU sent by $N_{0}$. Thus, $N_{0}$ has an alternate port through $N_{1}$. Similarly for $N_{1}$, it must have an alternate port to $R$ via another bridge, say $N_{2}$, and $N_{2}$ 's BPDU is superior to $N_{1}$ 's so $N_{1}$ has an alternate port through $N_{2}$. This argument applies till bridge $N_{k-1}$. However, since there is a finite number of bridges, $N_{k}$ must obtain an alternate port to $R$ via one of the bridges $N_{0}$ to $N_{k-2}$. However, this is impossible because $N_{k}$ 's BPDU is superior to the BPDUs from all other bridges. Thus, we have a contradiction.

Because there exists at least one bridge in the partition that does not contain the previous root that has no alternate port, by the RSTP protocol (rule (1)), this bridge, when it detects that its root port is no longer valid, it must declare itself the new root and begin sending BPDUs announcing itself the root. These BPDUs will be flood along the partition. The next lemma shows that if the partition contains a cycle, then there exists a race condition such that if the BPDU arrives at a bridge with an alternate port via its root port first, stale information cached at its alternate port about the previous root will be spread into the network, creating the count-to-infinity situation.

Lemma 2: If a network is partitioned, and the partition without the previous root bridge contains a cycle, a race condition exists that may lead to count-to-infinity which can create a temporary forwarding loop.

Proof: From Lemma 1, in the partition containing the cycle, one or more bridges without alternate port must eventually declare themselves as roots and send their own BPDUs to the rest of the bridges in the partition. In addition, before the partition, the cycle must contain one or more bridges with an alternate port to the root. This is because, before the partition, assuming no forwarding loop exists, the cycle must be cut in the active forwarding topology by RSTP. An alternate port therefore exists at the link where the cycle is cut.

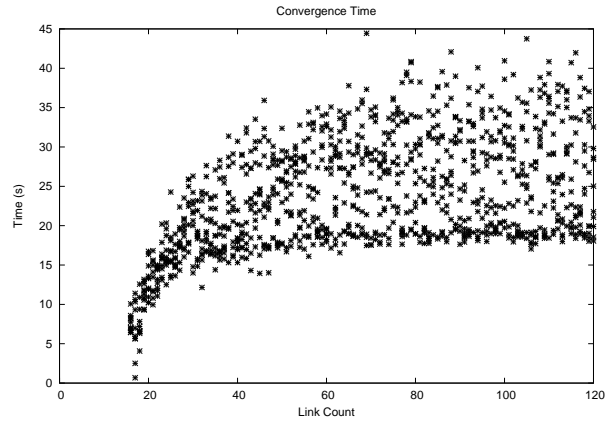

Fig. 5. Convergence time in a network of 16 bridges after failure of the root.

Now consider Figure 4 where the link between bridges i and $\mathrm{j}$ is where an alternate port exists in the cycle. Bridge $\mathrm{i}$ is connected to the rest of the loop with a root port on its left and has a designated port that links it to bridge $\mathrm{j}$. Bridge $\mathrm{j}$ is connected to the loop by its root port on its right and connected to bridge i by an alternate port. After the partition, BPDUs from bridges declaring themselves to be root will race around the cycle.

If bridge $\mathrm{j}$ receives such BPDUs on its root port before receiving them on its alternate port, it will find that its alternate port has superior cached information suggesting a path to a superior root that is no longer reachable. Thus bridge $\mathrm{j}$ will then make the alternate port its root port and start sending BPDUs conveying the information it has cached to bridges on its right as it believes it has superior information than the one it received. Afterwards, bridge $\mathrm{j}$ would get BPDUs on its new root port through bridge $\mathrm{i}$ from bridges declaring themselves to be root. Bridge $\mathrm{j}$ will then know that the information at its root port is stale and will accept the new information and also forward it to its right. This will result in a situation where fresh BPDUs chasing stale BPDUs around the loop resulting in a count-to-infinity situation and may create a temporary forwarding loop.

On the other hand if bridge $\mathrm{j}$ receives the fresh BPDUs from other bridges declaring themselves to be root on its alternate port first before receiving them on its root port, the stale information at the alternate port will be discarded and no count-to-infinity would occur.

Count-to-Infinity may even occur without a network partition. For example if the loop in the physical topology loses its cheapest path to the root and picks another path with a higher cost. This new information will race around the loop until it reaches an alternate port caching stale, but superior information. Again this stale information will chase the new information around the loop counting to infinity. This will keep going until the stale information reaches its MaxAge, or the cost reported by the stale information increases to exceed that of the new information. This is because the cost reported by the stale information increases while it is circling around the loop, counting to infinity.

In summary, the problem is bridges cache information from the past at alternate ports, then use it blindly in the future if the root port becomes invalid, without knowing whether this 


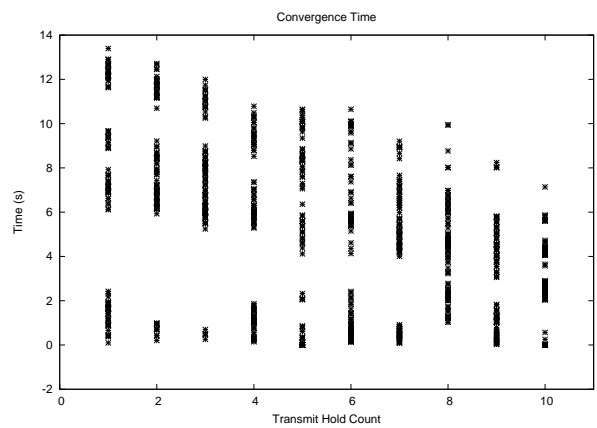

(a) Complete graph topology, 4 bridges.

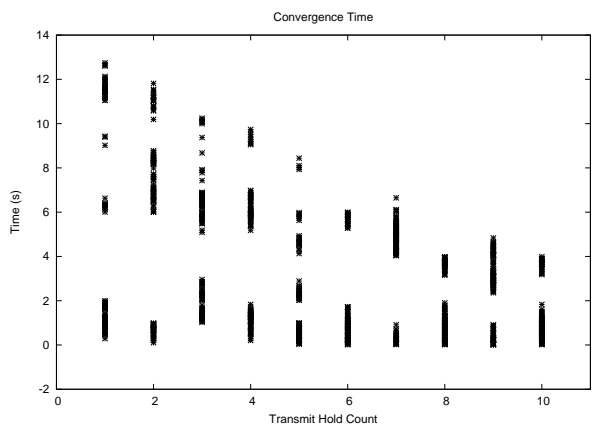

(b) "Loop" topology, 4 bridges.

Fig. 6. Convergence time after failure of the root varying the TxHoldCount.

information is stale or not. Then the bridge starts spreading this stale information to other bridges via its BPDUs potentially resulting in a temporary forwarding loop.

\section{Coping with Counting-to-Infinity in RSTP}

1) Reduce Max Age: In RSTP, BPDUs include a Message Age that is initialized to zero by the root. Every bridge that passes the BPDU along the network increments the BPDU's Message Age by one before forwarding it. When a BPDU's Message Age reaches MaxAge it gets dropped. The value of MaxAge prescribed by the standard is 20 . This results in stale information eventually getting flushed out of the network. Thus, one potential strategy to cope with the counting-toinfinity problem is to reduce MaxAge. Unfortunately, reducing MaxAge under the current RSTP standard also unnecessarily limits the network diameter and thus the scalability of Ethernet.

But much more importantly, the MaxAge parameter does not effectively bound the time counting-to-infinity can persist. One may think that count-to-infinity can last for at most a few milliseconds as the stale BPDUs are dropped after they traverse at most 20 hops around the loop. Unfortunately RSTP has a per port Transmit Hold Count (TxHoldCount) that limits the number of transmitted BPDUs per port per second. Each time a BPDU is transmitted through a port, the transmit count is incremented until it reaches the TxHoldCount, where no more BPDUs can be transmitted. The transmit count is decremented every clock tic, which occurs every one second. The purpose of such limit is to protect a bridge from being overwhelmed by processing a lot of BPDUs, specially if the bridge is serving multiple VLANs. The TxHoldCount exacerbates the count to infinity problem by delaying the transmission of the BPDUs. Depending on the complexity of the network topology, the volume of BPDUs will vary. Thus the time it takes for a BPDU to reach Max Age also varies with network complexity.

To illustrate this problem, we simulate a network of 16 bridges that is initially configured in a ring topology. Then we randomly add redundant links to increase complexity until we reach a fully connected graph. After adding each link we simulate the failure of the root bridge and measure the convergence time. What we mean by convergence time is the time, measured in seconds, after which all the bridges in the network have agreed on the same correct root bridge. Figure 5 shows that adding more redundant links dramatically increases the the convergence time. The reason for that is adding more redundant links results in more alternate ports per bridge. If the root bridge is unreachable and the bridge has many alternate ports, it may try all its alternate ports one after another. Every time a bridge switches to a new alternate port, this port goes forwarding triggering a topology change event that makes the bridge send topology change message on all its forwarding ports. For example in Figure 2(c) and (d), when bridge 4 takes its alternate port as its new root port, a topology change is triggered. Bridge 4 sends a topology change message on all its forwarding ports. Thus switching between a few alternate ports can result in all the ports in the active topology reaching their TxHoldCount limit due to the transmissions of all the topology change messages. This increases the convergence time as BPDU updates cannot be transmitted promptly.

2) Increase Transmit Hold Count: It follows that another potential way to cope with RSTP's counting-to-infinity is to increase the TxHoldCount at the expense of increasing the BPDU processing load on bridges. One may think that by increasing the TxHoldCount, the duration a stale BPDU can persist in a network should be proportionally reduced. Unfortunately, that is not the case in reality.

To illustrate, we simulate a fully connected network of 4 bridges and measure the convergence time after the death of the root bridge. Figure 6(a) shows the convergence times for ten runs when varying the TxHoldCount according to the value range allowed by the standard. We can see that the convergence time exhibits a multi-modal behavior. Even when the TxHoldCount is increased to 10 , the worst case convergence time is still 8 seconds, not the 10 times improvement one might expect when comparing to a TxHoldCount of 1 . Clearly, the benefit of increasing TxHoldCount is non-linear and limited. This is because once the transmit count reaches the TxHoldCount limit, it gets decremented by one every second allowing for only one BPDU to be transmitted per second irrespective of the TxHoldCount value. Figure 6(b) shows the measured convergence time for a simpler topology, 


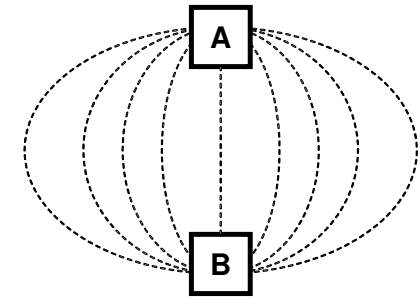

Fig. 7. A family of safe topologies of RSTP bridges

namely the topology in Figure 2(a). Even in this simple topology, increasing the TxHoldCount does not dramatically improve convergence time.

3) Use Restricted Topologies: Another potential way to cope with RSTP's counting-to-infinity is to avoid it by using restricted network topologies in which the problem cannot manifest. Since counting-to-infinity can happen when there is a cycle in the physical topology and this cycle gets partitioned from the root, topologies having physical cycles that can get partitioned from the root should be avoided when using RSTP bridges.

A safe topology therefore should have every cycle pass through the root bridge. A family of topologies that satisfy this requirement and is not prone to partitioning after the death of the root bridge is topologies having all their cycles intersect at two bridges in which one is the root bridge. Figure 7 is an example of such topologies. In this example the root should be selected among bridges $\mathrm{A}$ and $\mathrm{B}$. This can be done by manipulating the bridge's priority to guarantee that it has the lowest bridge ID in the network. A ring topology is a special case of such family of topologies.

\section{RSTP WITH EPOCHS: EXTENDING RSTP TO ELIMINATE THE COUNT TO INFINITY}

In this section, we introduce the RSTP with Epochs protocol that solves the count to infinity problem that we presented in Section III. The RSTP with Epochs protocol is an extension to the RSTP protocol [9] that relies on the root adding a sequence number to each BPDU that it generates. Designated bridges generate and transmit their own BPDUs based on the latest root's BPDU and including the root's latest sequence number. The purpose of these sequence numbers is to identify stale BPDUs or stale cached information from a retired root. However sequence numbers by themselves are not sufficient. For example, consider in a network of bridges where there is the old root bridge $\mathrm{A}$ and a new bridge $\mathrm{B}$ with lower bridge ID than A that has just joined the network. Bridge B is now eligible to become the root, so when it receives a BPDU from $\mathrm{A}$, it starts sending out its own using a sequence number higher than the one in A's BPDU. This is to override A's BPDUs and assert itself as the new root causing A to back-off. However, by the time B's BPDU reaches A, A may have sent out one or more BPDUs having higher sequence numbers. Thus $\mathrm{A}$ will view B's BPDUs as stale and it will not back off and the network will not converge.
Using epochs solves this problem. An epoch is an interval starting when the true root bridge achieves root status and ends with another bridge contending for root status. Another bridge will contend for root status because it did not hear from the previous root, or because it finds its bridge ID to be lower than that of the previous root. A bridge may not hear from the previous root if the previous root has retired, or the root may still be reachable but the contending bridge has lost its path to the root without having any other alternate ports. A bridge may find it has a lower bridge ID than the root because it has just joined the network and its bridge ID is lower than the current root's bridge ID, so it's eligible to be the new root. If the previous root has retired and the contending bridge is eligible to be the root, the new root will use a sequence number higher than the highest sequence number it received from the retired root signaling a new epoch with a new root bridge. If the old root is reachable and is still eligible to be the root, it pumps up its sequence number to override the contending bridges' sequence numbers to re-take the network and this signals a new epoch as well but with the same root bridge as in the previous epoch. Each bridge has a local representation of an epoch with an interval of sequence numbers it heard from the same root bridge. The interval is represented by two sequence numbers, FirstSeqno and CurrentSeqno. FirstSeqno is the first sequence number this bridge has heard from the current root. CurrentSeqno is the current or latest sequence number the bridge has heard from the root. Back to the example given above, epochs allow the new root B to catch up with the old root's sequence numbers to eventually be able to take over the network. When B's BPDU reaches A, A may have already sent BPDUs with higher sequence numbers, but since B's BPDU sequence number lies within the interval representing the current epoch, A realizes that B coexists with it in the same epoch and thus it backs away. Subsection IV-A presents the RSTP with Epochs protocol in details. Then subsection IV-B further discusses the operation of the protocol.

\section{A. Protocol Definition}

In detail, the RSTP with Epochs protocol modifies the RSTP protocol as follows:

1) The periodic BPDUs sent by the root have increasing sequence numbers (BPDU.Seqno), where the period is typically a HelloTime. The sequence number is incremented by the root bridge at the beginning of each period. Children bridges generate their BPDUs including the root's latest sequence numbers.

2) Each bridge records two values, FirstSeqno and CurrentSeqno, the first and last sequence numbers, respectively, that it has received from the current root bridge. These two sequence numbers define the current epoch. The purpose of this epoch is to identify stale BPDUs. A BPDU with a sequence number less than the recorded first sequence number must be a stale BPDU belonging to an earlier epoch.

3) Bridges disregard the sequence numbers when comparing BPDUs declaring the same root. However, if 


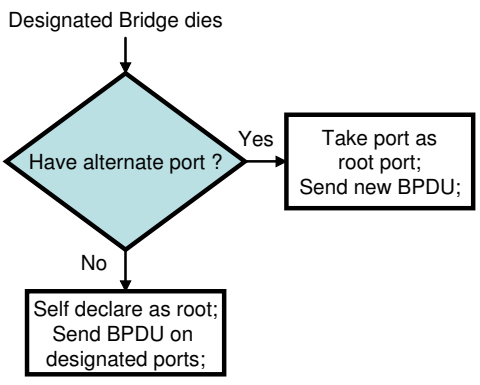

Fig. 8. Handling the death of the designated bridge

a BPDU arrives declaring a different root than the one perceived by the bridge, the bridge checks if the BPDU's sequence number is larger than the last recorded sequence number for the perceived root. If this is the case, it signals the beginning of a new epoch. The new epoch has a different root declared by the received BPDU. The first and last sequence numbers are set to the sequence number reported by the received BPDU. On the other hand, if the sequence number reported by the BPDU is larger than or equal to the first recorded sequence number but smaller than or equal to the largest recorded sequence number of the current root, the bridge with the lowest ID - among the ones declared by the BPDU and the current root - is deemed superior; and it is the one accepted by the bridge as the current root.

4) When a bridge detects disconnection from its designated bridge, it first checks to see if it has any alternate ports. If it does, it adopts one of these alternate ports as its new root port. However, if the bridge does not have any alternate ports, it declares itself as the new root and starts broadcasting its own BPDUs that have a sequence number larger than the last sequence number that it received from the old root.

5) If a bridge receives a BPDU declaring another bridge with an inferior bridge ID to its own as the root, the bridge starts sending BPDUs declaring itself as the root. These BPDUs are given a sequence number that is larger than that received from the inferior bridge. When one of these BPDUs reaches the inferior bridge, it will stop declaring itself as the root.

Figure 8 explains how a bridge handles the event of the death of its designated bridge; this is the same way an RSTP bridge handles this event. Figure 9 explains the handling of a the receipt of a BPDU for the RSTP with Epochs protocol.

\section{B. Discussion}

Sequence numbers can wrap around. The way to deal with that is to consider zero as bigger than the largest sequence number. A side effect of doing that is when a new bridge joins the network starting off with sequence number zero may be able to temporarily take over the network although it has a bridge ID higher than the legitimate root. When the legitimate root receives the new bridge's BPDU, it can then pump up its sequence number and re-take the network. This may result in a brief period of disconnectivity. A work around this problem is to make a new bridge joining the network listen for a while for BPDUs, if it receives a BPDU from a superior root, it should not send its own BPDU. If no superior BPDUs are received the new bridge can then start sending its own BPDU declaring itself to be the root.

The advantage of RSTP with Epochs when compared to RSTP is that it avoids count-to-infinity. On the other hand, its disadvantage when compared to RSTP is the small overhead that can result from its comparative pessimism. To elaborate, let us reconsider the topology in Figure 1. Suppose the link between bridge 2 and 3 dies. Under both protocols, bridge 3 will emit a new BPDU. The difference is, in RSTP, the propagation of this BPDU will be stopped once it reaches bridge 5 because bridge 5 has an alternate port to the root via bridge 6 . In effect, by default RSTP assumes that the root bridge is still alive. In contrast, in RSTP with Epochs, this BPDU creates a new epoch and thus is superior to the cached information at the alternate port at bridge 5. Consequently the propagation will not be stopped until it reaches bridge 1. In effect, RSTP with Epochs pessimistically assumes that the root bridge is inaccessible.

In absence of a count-to-infinity, both RSTP and RSTP with Epochs generate the same topology change events and thus generate the same number of BPDUs signaling topology change events. This is because a topology change event occurs when a port goes forwarding and since both protocols converge at the same topology, switching the same ports to forwarding and thus generating the same topology change events. In case of a count-to-infinity in RSTP, some ports may go to forwarding temporarily generating some extra topology change events as in Figure 2.

\section{Interoperability with Legacy Bridges}

In this section, we provide some suggestions on how to allow RSTP with Epochs bridges to interoperate with legacy RSTP and STP bridges. The basic mechanism is similar to that used by RSTP to interoperate with STP. First, the RSTP with Epochs protocol should be assigned a new protocol version number. A BPDU sent by a bridge carries the version number of the corresponding protocol used. A BPDU with an unknown version number will be discarded by the receiving bridge. At start up, a RSTP with Epochs bridge will try sending RSTP with Epochs BPDUs. If the network peer is a legacy bridge, these BPDUs will be ignored. Eventually, the RSTP with Epochs bridge will receive legacy BPDUs from the legacy peer bridge, at such time it can recognize the protocol used by the peer and fall back to the appropriate legacy protocol. To translate a RSTP with Epochs BPDU into a legacy BPDU, the epoch sequence number is simply stripped from the BPDU. These mechanisms allow a mixture of RSTP with Epochs, RSTP, and STP bridges to co-exist in a network.

A careful design of the network can also help to extract the most benefits from RSTP with Epochs bridges even when they are mixed with legacy bridges. First, redundancy is most 


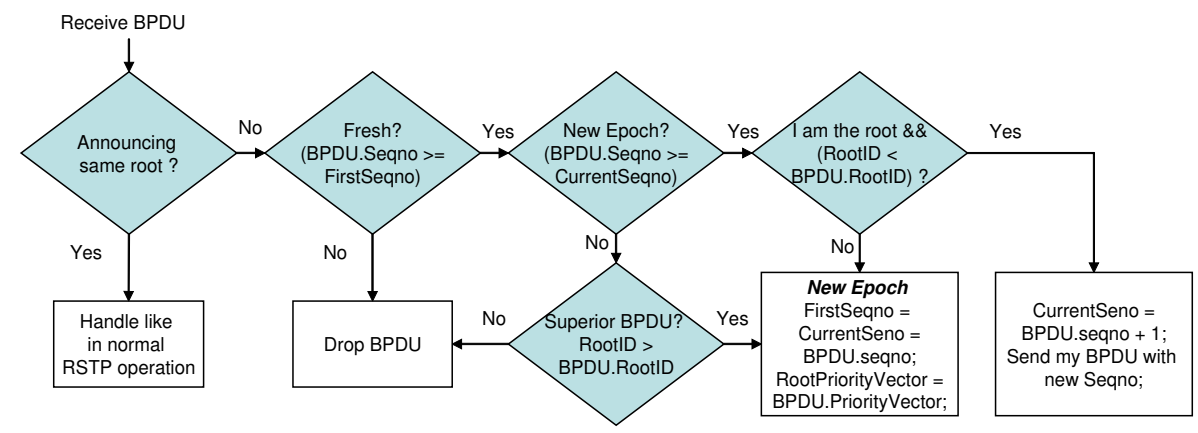

Fig. 9. Handling the reception of a BPDU in the RSTP with Epochs protocol

critical in the core of the network. Thus, RSTP with Epochs bridges should be used in the network core, where many redundant links can be safely introduced. Legacy bridges can be used as traffic aggregation trees at the edge of the network. These aggregation trees contain no cycles and thus are safe. To ensure an RSTP with Epochs bridge will be elected as the new root upon a failure, RSTP with Epochs bridges should be assigned the lowest IDs among all bridges in the network.

\section{Evaluation}

In this section we evaluate different aspects of the the RSTP and the RSTP with Epochs protocols. Section V-A presents our evaluation methodology. Then, in Section V-B the convergence times of both protocols are evaluated. The packet overhead of both protocols is studied in Section V-C. Finally in Section V-D we study how counting to infinity can saturate a bridge's maximum BPDU transmission rate limit (i.e. the TxHoldCount), thus preventing the timely announcements of other BPDUs.

\section{A. Evaluation Methodology}

To evaluate RSTP and RSTP with Epochs protocols we used the simulator used by [11]. We extended it to include the RSTP with Epochs implementation, to have desynchronized bridge clocks, and also added some instrumentations to allow us to collect information required in our experiments. The simulator uses a MaxAge value of 20, HelloTime of 2 seconds and a TxHoldCount of 3 unless otherwise stated. Not all bridges start together at time zero. Instead each bridge starts with a random offset from time zero that is a fraction of the HelloTime. Bridges are connected to each other by links with 100 microsecond of total delay (propagation and transmission delay). Only protocol BPDU packets are simulated. No user data packet traffic is simulated.

\section{B. Comparing Convergence Times of Both Protocols in Event of Failure}

In this subsection we compare the convergence times of RSTP and RSTP with Epochs in the event of failure in three families of topologies. What we mean by convergence time is the time it takes the network until all its bridges have converged to the correct active topology. For each family of topologies we vary the number of bridges in the network and measure the corresponding convergence time. For each data point we repeat the experiment 100 times and report the range of values measured.

In the first experiment we simulate a set of complete graphs, varying the number of bridges in the network. In each run we kill the root bridge and measure the time it takes for the network to converge under both protocols. Figure 10 shows the convergence times measured. It presents bars representing the range of values measured for each network size. The $\mathrm{x}$-axis is shifted downward to show that the convergence times for RSTP with Epochs is negligible compared to those of RSTP. In fact the highest convergence time observed for the RSTP with Epochs protocol is only 100 microseconds. This is because RSTP with Epochs does not suffer from the count to infinity problem and its convergence is only limited by the inherent network delay. On the other hand, RSTP takes much longer to converge. The variance in the convergence times for RSTP is due to the variability in the race conditions when count to infinity occurs.

In the second set of experiments we use simpler "loop" topologies, similar to the topology in Figure 2(a) where we vary the total number of bridges in the loop. For example, a network with 10 bridges means the loop has 9 bridges and the loop is connected to the root bridge that does not lie on the loop. Like in the previous experiment we kill the root bridge and measure the convergence time for both protocols. Figure 11 shows the convergence times measured. Again, RSTP with Epochs can converge in at most 400 microseconds in these experiments, but RSTP takes seconds to converge even under this simple network setting.

In the third set of experiments we use simple "ring" topologies where the bridges form a simple cycle. We take down the link connecting the root bridge bridge $(\mathrm{R})$ to a neighbor bridge (N). In RSTP, since $\mathrm{N}$ does not have any alternate ports, it will declare itself as root and start broadcasting its BPDU, the BPDU will flow through its descendants, invalidating the information at their root ports, until it reaches a bridge with an alternate port to the root. Since the alternate port caches superior information, the bridge will pick the alternate port as its root port and will send this new information back to $\mathrm{N}$ so it will eventually know that $\mathrm{R}$ is alive and accept it as its 


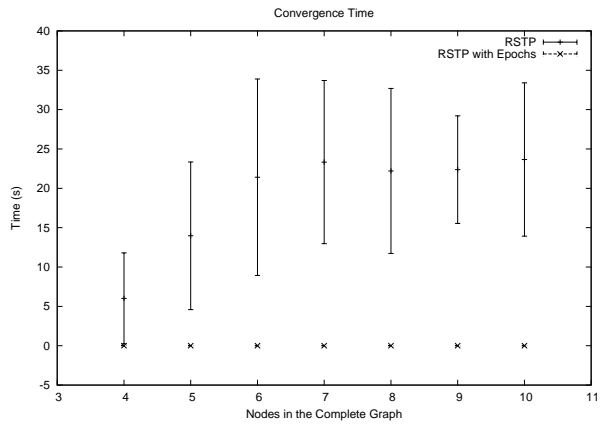

Fig. 10. Convergence time after failure of the root in complete graph topologies of 4 to 10 bridges. Each experiment is run 100 times and the range of convergence times is shown.

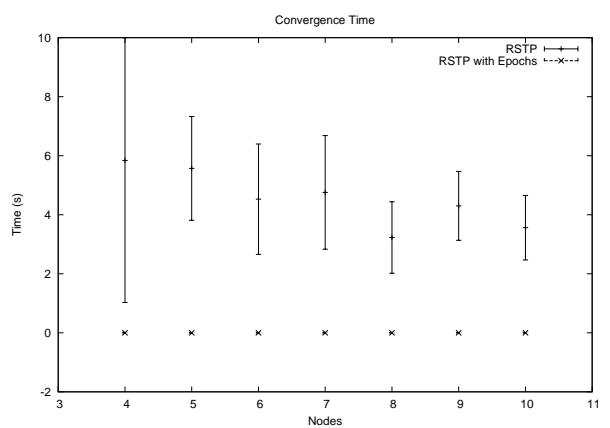

Fig. 11. Convergence time after failure of the root in "loop" topologies of 4 to 10 bridges. Each experiment is run 100 times and the range of convergence times is shown.

root. This means that N's BPDU will travel half way around the ring to reach the bridge with the alternate port, then the bridge with the alternate port will send a BPDU that will travel back to $\mathrm{N}$, until $\mathrm{N}$ knows that $\mathrm{R}$ is alive.

Conversely in RSTP with Epochs, $\mathrm{N}$ will detect disconnection from the root, so it will send a BPDU with a higher sequence number than the last BPDU it has received from the root $\mathrm{R}$. This will signal a new epoch to all bridges in the ring and they will accept N's BPDU as it has higher sequence number. Eventually N's BPDU will reach R after traveling all the way around the loop. R, knowing it is the legitimate root, will in response increase its sequence number and send a new BPDU to assert itself as the root. R's BPDU with the higher sequence number will make its way to $\mathrm{N}$ after traveling all the way back around the network which will make $\mathrm{N}$ accept $\mathrm{R}$ as its root.

The effect of these different behaviors can be observed in Figure 12 where RSTP with Epochs takes roughly twice the amount of time to converge compared to RSTP. Note that the convergence times for both protocols are very small in these experiments. In this experiment there is no variance in the results as there are no race conditions and thus the results are deterministic.

\section{Comparing BPDU Overhead of Both Protocols}

In this section we present experiments that illustrate the BPDU overheads of both RSTP and RSTP with Epochs protocols using the three families of topologies as used in Section V-

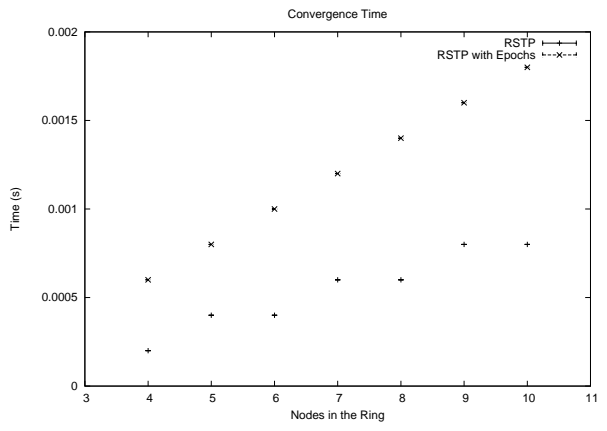

Fig. 12. Convergence time after failure of a link connected to the root in ring topologies of 4 to 10 bridges. Each experiment is run 100 times and the range of convergence times is shown.

B. In this set of experiments we present histograms plotting the total number of packets transmitted in the network within every tenth of a second. We exclude the packets transmitted to or from the root bridge as the root bridge dies at time 20 and we want to factor out the effects of having different number of bridges in the network before and after the death of the root bridge. Each histogram presents the packet transmissions in the network in a single experiment run.

In the first experiment we simulate a complete graph of 10 nodes. We kill the root bridge at time 20. Figure 13(a) and Figure 13(b) show the histograms of BPDUs transmitted for the RSTP and the RSTP with Epochs protocols respectively during a 100 second time span. For both protocols we observe a spike in the BPDUs transmitted at startup time. This is because at startup each bridge sends out its BPDU and keeps sending out any new superior information it receives until the bridges in the network agree on the same root and converge to the final spanning tree. After that the network goes into steady state where bridges only send the periodic hello message every hello time. At time 20, the root bridge dies. RSTP suffers from the count to infinity problem and sends out a lot of packets during a time span that exceeds 25 seconds until the network converges. RSTP with Epochs reacts differently to the failure of the root. There is an initial spike in the packets transmitted as the new information - of the death of the root and a new bridge asserting itself as the new root - flows throughout the network. Then the network converges almost instantaneously and BPDU transmission returns to steady state.

In the second experiment we simulate a topology similar to that in Figure 2(a) with 10 bridges, 9 of them are in the loop. We kill the root bridge at time 20. Figure 14(a) and Figure 14(b) show the histograms of BPDUs transmitted for the RSTP and the RSTP with Epochs protocols respectively during a 100 second time span. Again, for both protocols we observe a spike in the BPDUs transmitted at startup time. After that the network goes into steady state where bridges only send the periodic hello message every hello time. At time 20, the root bridge dies. Similar to the first experiment RSTP suffers from the count to infinity problem and sends out a lot of packets until the network converges. RSTP with Epochs converges almost instantaneously requiring 


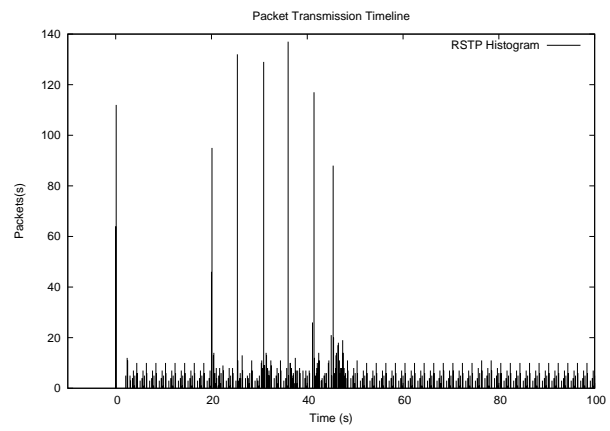

(a) RSTP protocol.

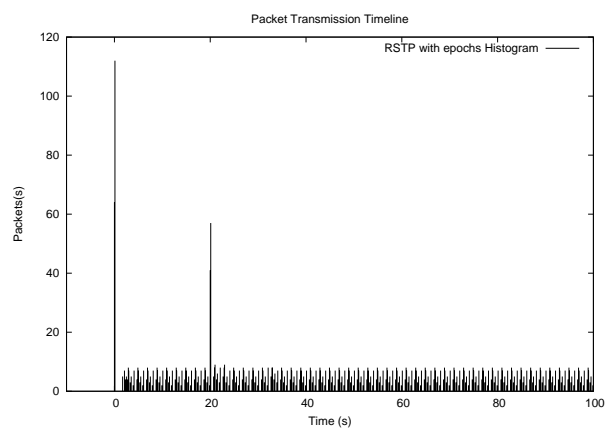

(b) RSTP with Epochs.

Fig. 13. Histogram of BPDU packet transmissions in a 10 bridge fully connected graph topology, each bin is 0.1 second. The root bridge dies at time 20 .

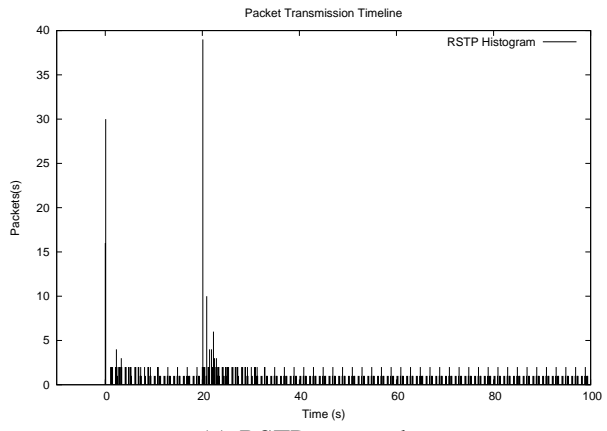

(a) RSTP protocol.

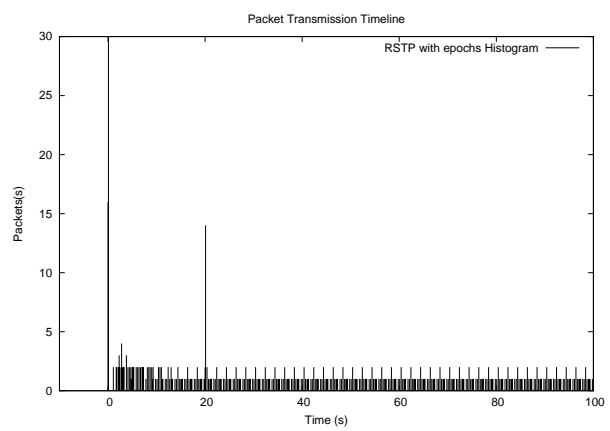

(b) RSTP with Epochs.

Fig. 14. Histogram of BPDU packet transmissions in a 10 bridge "loop" topology, each bin is 0.1 second. The root bridge dies at time 20 .

much fewer BPDUs to converge.

In the third experiment we simulate a 10 bridge ring topology. Similarly, we kill the link connecting the root bridge to a neighbor at time 20. Figure 15(a) and Figure 15(b) show the histograms of BPDUs transmitted for the RSTP and the RSTP with Epochs protocols respectively during a 100 second time span. In this experiment we observe that RSTP with Epochs uses more BPDUs than RSTP to recover from the failure. This because as explained in Section V-B, in RSTP with Epochs the disconnected bridge sends BPDU that traverses more hops than that in the case of the RSTP protocol.

In the three sets of experiments we note a short period of time after convergence where there is higher rate of packets being transmitted. This is because of the topology change events that result in an extra BPDU getting transmitted through each bridge's root port every HelloTime and this lasts during the duration of the topology change timer.

\section{Effect of Counting to Infinity on Port Saturation}

In this subsection we study port saturation of both protocols in the event of failure using the three families of topologies as used in the previous experiments. A port is said to be saturated if it has reached its TxHoldCount limit but still has more BPDUs to transmit. We present a time sequence of the number of saturated ports in the whole network in the three experiment scenarios presented in Section V-C.

In the first experiment simulating a complete graph of
10 nodes we observe in Figure 16 a spike in the number of saturated ports at startup due to the spike in transmitted BPDUs at startup by both protocols. However starting from time 20 when the root port dies, we find a long period of time that is close to 20 seconds in the RSTP protocol where the network has many saturated ports. This is due to the count to infinity problem where BPDUs spin around the loop causing the ports to quickly reach their TxHoldCount limit. RSTP with Epochs does not suffer from the count to infinity problem, thus the ports do not get saturated after the failure.

Similarly, in the second experiment - simulating a topology like that in Figure 2(a) with 10 bridges - we observe in Figure 17 a spike in the number of saturated ports at startup. We also observe in the RSTP protocol a period after the failure of the root bridge where there are several saturated ports. Again this is because of the count to infinity problem.

In the third experiment simulating a ring topology, failure of the root cuts the loop so there is no count to infinity. Thus for both protocols no ports get saturated after the failure as can be seen in Figure 18.

\section{RELATED WORK}

The count-to-infinity behavior of RSTP was mentioned in [11]. However no thorough analysis was provided on the problem and its causes and no solution was provided. To the best of our knowledge no careful examination of this problem exists in the literature. Perlman proposed Rbridges [14], which 


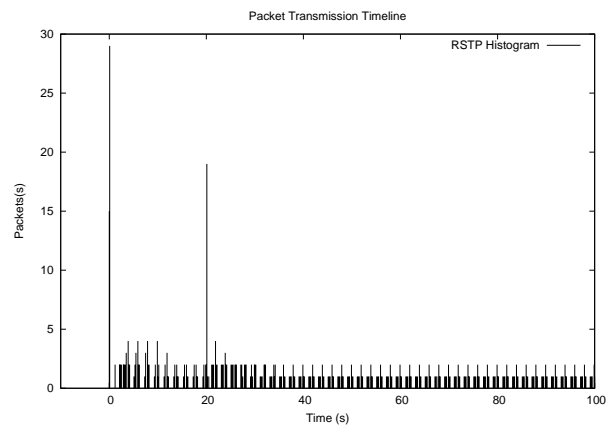

(a) RSTP protocol.

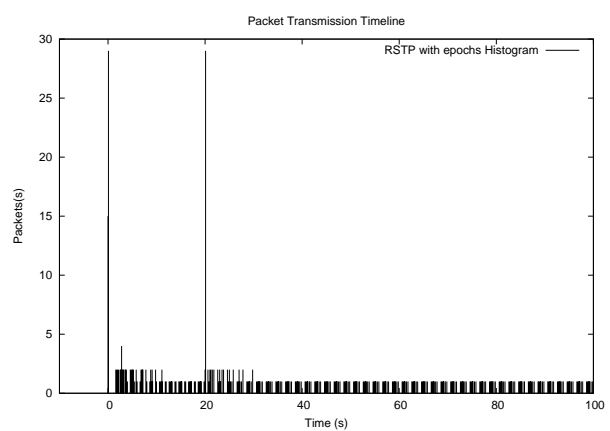

(b) RSTP with Epochs.

Fig. 15. Histogram of BPDU packet transmissions in a 10 bridge ring topology, each bin is 0.1 second. A link connecting the root bridge to a neighbor dies at time 20 .

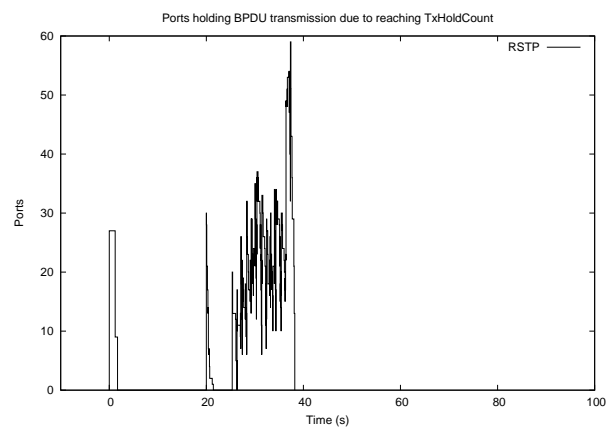

(a) RSTP protocol.

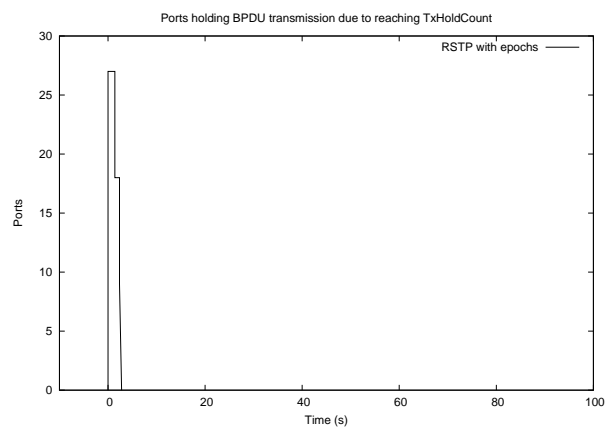

(b) RSTP with Epochs.

Fig. 16. Time sequence of number of ports that have reached their TxHoldCount limit while they still have more BPDUs waiting for transmission. This experiment is for a 10 bridge fully connected graph topology where the root bridge dies at time 20 .

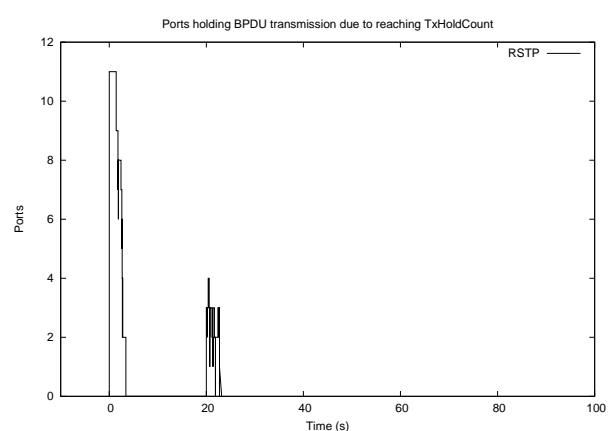

(a) RSTP protocol.

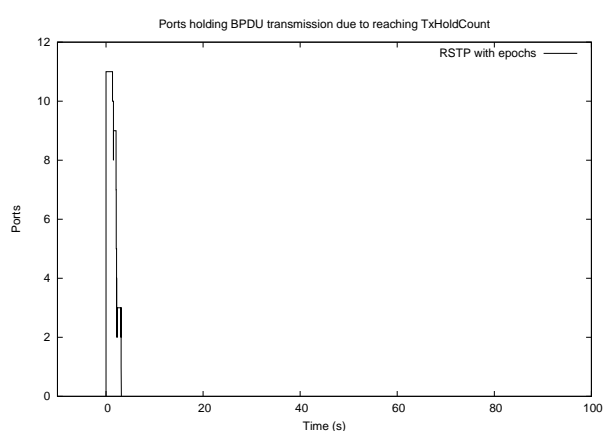

(b) RSTP with Epochs.

Fig. 17. Time sequence of number of ports that have reached their TxHoldCount limit while they still have more BPDUs waiting for transmission. This experiment is for a 10 bridge "loop" topology. The root bridge dies at time 20.

employ link state routing among Rbridges rather than relying on spanning trees. The way Rbridges deals with routing loops is by including a time to live field in all packets. It achieves that by encapsulating all packets adding its own header. Garcia et al. proposed replacing the spanning tree with link state routing as well [6], however they do not provide a mechanism to deal with temporary routing loops. SmartBridge [15] uses complex internodal coordination mechanism, namely diffusing computations [4], to achieve effective global consistency and consequently loop-freeness. SmartBridges require freezing the network and discarding all the data during convergence time after a topology change.

Pellegrini et al. propose a different technique to break cycles other than the spanning tree protocol. Their technique is based on turn-prohibition [12]. Sharma et al. [16] introduce a multispanning tree architecture that improves the throughput and reliability over when using a single spanning tree.

STP and RSTP implement a variant of Distance Vector (DV) routing. RSTP with Epochs extends RSTP to eliminate the count-to-infinity problem. Other variants of DV routing have been proposed in the literature that are loop-free. For example [7], [10], [8] employ diffusing computations as well when 


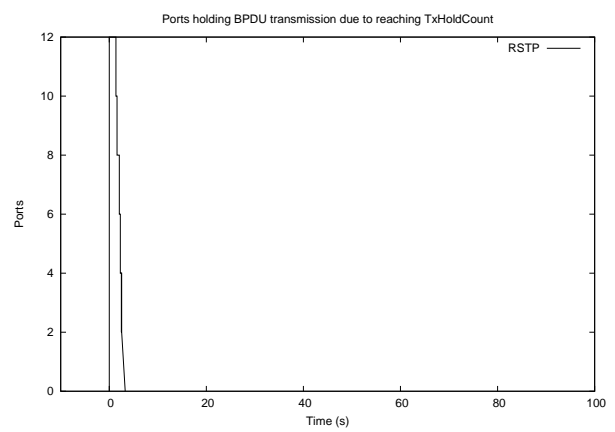

(a) RSTP protocol.

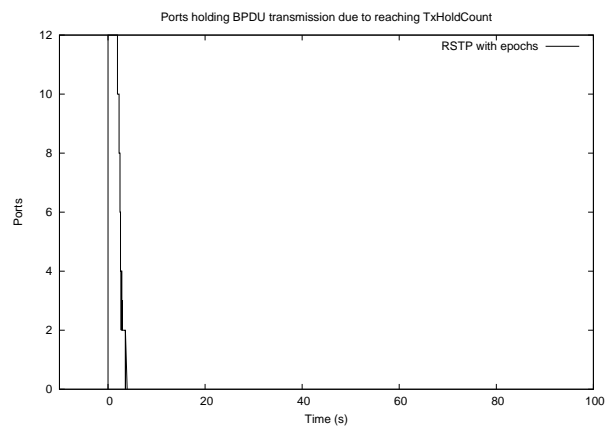

(b) RSTP with Epochs.

Fig. 18. Time sequence of number of ports that have reached their TxHoldCount limit while they still have more BPDUs waiting for transmission. This experiment is for a 10 bridge ring topology where a link connecting the root bridge to a neighbor dies at time 20 .

they make modifications to their routing tables to guarantee that their modifications are correct. This requires complex internodal coordination mechanisms. Perkins et al. proposed Destination-Sequenced Distance-Vector (DSDV) [13], where every node in the network periodically advertises a monotonically increasing sequence number. The latest sequence number received from a destination is included in its route information in the routing table. A route with a higher sequence number is always preferred over another route to the same destination with a lower sequence number. This is similar to RSTP with Epochs except that in RSTP with Epochs there is only one sequence number that is modified by the root bridge. If the root bridge retires the sequence number is inherited by the new root bridge. Also RSTP with Epochs only considers sequence numbers across the boundary of two epochs. Within the same epoch sequence numbers are not considered.

The use of epochs is not new, it has been used before in the literature, for example in [1], [5]. Birman et al. [1] include epoch number in messages to control the order of delivery of messages. Elnozahy et al. [5] call an epoch as an incarnation number though, where it is used to identify stale messages.

\section{CONCLUSIONS}

In studying RSTP under network failures, we find that it can exhibit a count-to-infinity problem which may lead to a temporary forwarding loop. Although RSTP intends to use the MaxAge parameter to limit the lifetime of stale protocol information in the network, the outcome turns out to be very unpredictable. This is caused by a variety of reasons: variability in the outcomes of the different race conditions in the network, the effect of network complexity on the number of alternate paths and on the volume of BPDU transmissions, and the effect of the TxHoldCount parameter in slowing down the propagation of both fresh and stale BPDUs. Increasing the TxHoldCount also does not lead to proportional improvement in convergence time. Ultimately, parameter tuning does not eliminate the fundamental problem with count-to-infinity in RSTP. By adding a simple sequence number to BPDU messages and slightly modifying the procedure of BPDU processing, we show that RSTP with Epochs is able to eliminate the count-to-infinity problem and achieve convergence time on the order of one round-trip-time across the network. This solution can therefore significantly enhance the dependability of Ethernet networks.

\section{REFERENCES}

[1] K. Birman, A. Schiper, and P. Stephenson. Lightweight causal and atomic group multicast. ACM Transactions on Computer Systems, 9(3):272-314, August 1991.

[2] Cisco Systems, Inc. Spanning Tree Protocol Problems and Related Design Considerations. At http://www.cisco.com/warp/public/473/16.html

[3] Cisco Systems, Inc. Understanding Rapid Spanning Tree Protocol (802.1w). At http://www.cisco.com/warp/public/473/146.html.

[4] E. W. Dijkstra and C. S. Scholten. Termination detection for diffusing computations. Information Processing Letters, 11(1):14, August 1980.

[5] E. Elnozahy and W. Zwaenepoel. Manetho: Transparent RollbackRecovery with Low Overhead, Limited Rollback and Fast Output Commit. IEEE Transactions on Computers, Special Issue on FaultTolerant Computing, pages 526-531, May 1992.

[6] R. Garcia, J. Duato, and F. Silla. LSOM: A link state protocol over mac addresses for metropolitan backbones using optical ethernet switches. In Second IEEE International Symposium on Network Computing and Apllications (NCA '03), 2003.

[7] J. J. Garcia-Lunes-Aceves. Loop-Free Routing Using Diffusing Computations. IEEE/ACM Transactions on Networking, 1(1):130-141, February 1993.

[8] J. M. Jaffe and F. H. Moss. A Responsive Distributed Routing Algorithm for Computer Networks. IEEE Transactions on Communications, 30:1758-1762, July 1982.

[9] LAN/MAN Standards Committee of the IEEE Computer Society. IEEE Standard for Local and metropolitan area networks: Media Access Control (MAC) Bridges - 802.1D, 2004.

[10] P. M. Merlin and A. Segall. A Failsafe Distributed Routing Protocol. IEEE/ACM Transactions on Networking, 27:1280-1287, September 1979.

[11] A. Myers, T. E. Ng, and H. Zhang. Rethinking the Service Model: Scaling Ethernet to a Million Nodes. In Third Workshop on Hot Topics in networks (HotNets-III), Mar. 2004.

[12] F. D. Pellegrini, D. Starobinski, M. G. Karpovsky, and L. B. Levitin. Scalable cycle-breaking algorithms for gigabit Ethernet backbones. In IEEE Infocom 2004, Mar. 2004.

[13] C. E. Perkins and P. Bhagwat. Highly dynamic Destination-Sequenced Distance-Vector routing (DSDV) for mobile computers. In ACM SIGCOMM 1994, pages 234-244, Aug. 1994.

[14] R. Perlman. Rbridges: Transparent routing. In IEEE Infocom 2004, Mar. 2004.

[15] T. L. Rodeheffer, C. A. Thekkath, and D. C. Anderson. SmartBridge:A scalable bridge architecture. In ACM SIGCOMM 2000, Aug. 2000.

[16] S. Sharma, K. Gopalan, S. Nanda, and T. Chiueh. Viking: A multispanning-tree Ethernet architecture for metropolitan area and cluster networks. In IEEE Infocom 2004, Mar. 2004. 